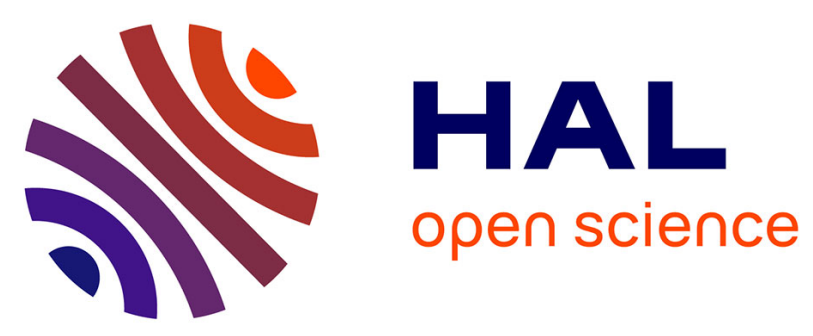

\title{
Thermally stable and electrically conductive, vertically aligned carbon nanotube/silicon infiltrated composite structures for high-temperature electrodes
}

Qi Ming Zou, Lei Min Deng, da Wei Li, Yun Shen Zhou, Hossein Rabiee Golgir, Kamran Keramatnejad, Li Sha Fan, Lan Jiang, Jean-François Silvain, Yong Feng Lu

\section{To cite this version:}

Qi Ming Zou, Lei Min Deng, da Wei Li, Yun Shen Zhou, Hossein Rabiee Golgir, et al.. Thermally stable and electrically conductive, vertically aligned carbon nanotube/silicon infiltrated composite structures for high-temperature electrodes. ACS Applied Materials \& Interfaces, 2017, 9 (42), pp.37340-37349. 10.1021/acsami.7b12087 . hal-01652001

\section{HAL Id: hal-01652001 https://hal.science/hal-01652001}

Submitted on 10 Mar 2021

HAL is a multi-disciplinary open access archive for the deposit and dissemination of scientific research documents, whether they are published or not. The documents may come from teaching and research institutions in France or abroad, or from public or private research centers.
L'archive ouverte pluridisciplinaire $\mathbf{H A L}$, est destinée au dépôt et à la diffusion de documents scientifiques de niveau recherche, publiés ou non, émanant des établissements d'enseignement et de recherche français ou étrangers, des laboratoires publics ou privés. 


\section{Thermally Stable and Electrically Conductive, Vertically Aligned Carbon Nanotube/Silicon Infiltrated Composite Structures for High-Temperature Electrodes}

Qi Ming Zou, ${ }^{\dagger, \pm}$ Lei Min Deng, ${ }^{\dagger \pm}$ Da Wei Li, ${ }^{\dagger}, *$ Yun Shen Zhou, ${ }^{\dagger}$ Hossein Rabiee Golgir,${ }^{\dagger}$

Kamran Keramatnejad, ${ }^{\dagger}$ Li Sha Fan, ${ }^{\dagger}$ Lan Jiang, ${ }^{\ddagger}$ Jean-Francois Silvain, ${ }^{\S}$ and Yong Feng Lu ${ }^{\dagger, *}$

${ }^{\dagger}$ Department of Electrical and Computer Engineering, University of Nebraska-Lincoln, Lincoln, NE 68588-0511, United States

${ }^{\mathfrak{E}}$ School of Mechanical Engineering, Beijing Institute of Technology, Beijing, 100081, China

${ }^{\S}$ Institut de Chimie de la Matière Condensée de Bordeaux, Avenue du Docteur Albert

Schweitzer F-33608 Pessac Cedex, France

KEYWORDS: Vertically aligned carbon nanotube, ceramic composite, infiltration, thermal chemical vapor deposition, high temperature electrode

ABSTRACT: Traditional ceramic-based, high-temperature electrode materials (e.g., lanthanum chromate) are severely limited due to their conditional electrical conductivity and poor stability under harsh circumstances. Advanced composite structures based on vertically aligned carbon nanotubes (VACNTs) and high-temperature ceramics are expected to address this grand challenge, in which ceramic serves as a shielding layer protecting the VACNTs from the oxidation and erosive environment, while the VACNTs work as a conductor. However, it is still a great challenge 
to fabricate VACNT-ceramic composite structures due to the limited diffusion of ceramics inside the VACNT arrays. In this work, we report on the controllable fabrication of infiltrated (and noninfiltrated) VACNT/silicon composite structures via thermal chemical vapor deposition (CVD) [and laser-assisted CVD]. In laser-assisted CVD, low-crystalline silicon ( $\mathrm{Si}$ ) was quickly deposited at the VACNT subsurfaces/surfaces followed by the formation of high-crystalline Si layers, thus resulting in noninfiltrated composite structures. Unlike laser-assisted CVD, thermal CVD activated the precursors inside and outside the VACNTs simultaneously, which realized uniform infiltrated VACNT-Si composite structures. The growth mechanisms for infiltrated and noninfiltrated VACNT-ceramic composites, which we attributed to the different temperature distributions and gas diffusion mechanism in VACNTs, were investigated. More importantly, the as-farbicated composite structures exhibited excellent multifunctional properties, such as excellent antioxidative ability (up to $1100{ }^{\circ} \mathrm{C}$ ), high thermal stability (up to $1400{ }^{\circ} \mathrm{C}$ ), good high velocity hot gas erosion resistance, and good electrical conductivity $(\sim 8.95 \mathrm{~S} / \mathrm{m}$ at $823 \mathrm{~K})$. The work presented here brings a simple, new approach to the fabrication of advacned composite structures for hot electrode applications. 


\section{INTRODUCTION}

High-temperature electrode applications require the electrode materials to be thermally stable, chemically inert, and electrically conductive and to possess a certain level of anticorrosive capability to high velocity hot gases. Traditional materials for high-temperature electrodes mainly include conducting ceramics $\left(\right.$ e.g., $\left.\mathrm{LaCrO}_{3}\right),{ }^{1}$ alumina-based ceramics, ${ }^{2-4}$ zirconia-based materials, ${ }^{5,6}$ and other oxides (e.g., magnesia ${ }^{2,7}$ and titanium oxide ${ }^{8,9}$ ). Due to the extreme properties required for the high-temperature electrode application, it is exceptionally challenging, even impossbile, for these single-compound electrode materials to satisfy all of the requreiments. For example, $\mathrm{LaCrO}_{3}$ as a solid oxide fuel cell electrode generates a large amount of heat during operation, ${ }^{10,11}$ which causes the operating temperature to be significantly elevated. Most of the oxide ceramics are insulators and are only conductive under certain conditions. ${ }^{2}$ On the other hand, novel electrodes, such as thin film electrode materials, have recently become popular. ${ }^{12-16}$ They are, however, not thermally stable when the operating temperature is higher than $1000{ }^{\circ} \mathrm{C}$. Considering the various limitations of these single-compound materials, by combining two or more materials with different physical and chemical properties to form an integrated structure, such as metal/ceramic composite structures, may achieve the required functionalities, as mentioned above.

Carbon-based ceramic composites are drawing much attention today due to their multifunctional properties, such as good electrical conductivity, strong mechanical strength, ${ }^{17,18}$ and good antioxidative ability (up to $650{ }^{\circ} \mathrm{C}$ ). ${ }^{18,19}$ Carbon nanotube $(\mathrm{CNT}) /$ ceramic composites, in particular, have demonstrated excellent properties in many aspects, especially good mechanical and excellent 
electrical properties. ${ }^{20}$ To achieve better electrical and thermal conductivities in CNT/ceramic composites, vertically aligned carbon nanotubes (VACNTs) based ceramic composites are preferrable due to the conductivity anisotropy of the VACNT conducting network along the axial direction. ${ }^{21}$ Common ceramics used in VACNT/ceramic composites include silicon carbide $(\mathrm{SiC}),{ }^{22}$ graphite, ${ }^{23}$ alumina,${ }^{24}$ mullite, ${ }^{25}$ silicon $(\mathrm{Si}),{ }^{14,26}$ and various nitrides. ${ }^{27,28}$ However, the existing VACNT/ceramic composites have poor antioxidative capabilities, making them unsuitable for high-temperature electrode applications. To address this issue, ceramics are required to be uniformly and densely deposited both inside and outside the VACNTs, forming infiltrated VACNT/ceramic composites, which can protect VACNT arrays from oxidation, corrosion, and erosion under the extreme harsh circumstances. However, achieving a uniform mixture of different phases remains a big challenge, especially for bulk VACNTs due to their high aspect ratio. ${ }^{20}$ As we know, the average intertube distance in VACNT arrays (without any pretreatments) is less than $10 \mathrm{~nm} .{ }^{29}$ In such high-density VACNT arrays, once a layer of ceramic thin film forms on the top surface of the VACNTs, ceramics will stop growing inside the VACNTs due to the blocking effect of the densely formed ceramic films. Several studies have been demonstrated the ability to successfully achieve CNT-ceramic composites through the incorporation of relatively low-density CNTs in ceramic matrices using sintering ${ }^{30}$ or hot pressing technique. ${ }^{31}$ However, in these composites, CNTs were used as reinforcement dispersed in a matrix instead of using their unique dimensionality and the desired properties (such as electrical and mechanical anisotropies in VACNTs). Recently, it has been reported that atomic layer deposition (ALD) is effective to obtain uniform infiltration of ceramics $\left(\mathrm{Al}_{2} \mathrm{O}_{3}\right)$ in $\mathrm{O}_{2}$-plasma-treated VACNT arrays. ${ }^{32}$ Despite some 
progress made so far, the direct infiltration of ceramics into dense VACNTs arrays without any pretreatments is still a big challenge. We believe that chemical vapor infiltration via thermal chemical vapor deposition (CVD) will allow simultaneous growth of ceramics from inside to outside of VACNTs, resulting in the fabrication of highly uniform and dense VACNT-ceramic composites.

In this work, we have, for the first time, developed an efficient approach to controllable fabrication of infiltrated (and noninfiltrated) bulk VACNT/ceramic (Si or gallium nitride (GaN)) composite structures via a single-step thermal CVD [and laser-assisted CVD] (Figure 1a). Vertically aligned carbon nanotubes, but not other carbon nanomaterials, were chosen for the conducting network and building framework, as VACNTs minimize the thermal stress at elevated temperatures. ${ }^{33}$ The growth mechanisms for infiltrated and noninfiltrated VACNT-ceramic composites were investigated, which we attributed to the different temperature distributions and the gas diffusion mechanism in VACNTs during thermal CVD and laser-assisted CVD processes. The as-prepared infiltrated VACNT-Si composite structures exhibited excellent high-temperature antioxidative ability (up to $1100{ }^{\circ} \mathrm{C}$ ), high thermal stability (up to $1400{ }^{\circ} \mathrm{C}$ ), and good electrical conductivity and erosion resistance, suggesting that VACNT-Si composite structures have great potential for future high-temperature electrode applications under extreme conditions.

\section{METHODS}

Fabrication of ultralong VACNTs via thermal CVD: Ultralong VACNTs were fabricated using a thermal CVD method (Figure 1a). First, iron/aluminum oxide $\left(\mathrm{Fe} / \mathrm{Al}_{2} \mathrm{O}_{3}\right)(2 / 20 \mathrm{~nm})$ 
catalyst films were deposited onto a silicon dioxide $\left(\mathrm{SiO}_{2}\right) / \mathrm{Si}$ substrate $(2 \mu \mathrm{m}$ oxides $)$ in a magnetron sputtering system (AJA International, Inc., ATC-Orion 5 UHV). Then, the growth of VACNTs was carried out in a two-zone CVD furnace (MTI Corporation, OTF-1200X) (Figure S1a, Supporting Information). The growth parameters were similar to the work presented by $\mathrm{Wu}$ et al.. ${ }^{34}$ Two different types of VACNT samples were prepared: bulk VACNTs and cubic-patterned VACNTs.

Fabrication of VACNT-Si infiltrated composite structures: Infiltrated VACNT-Si composite structures were prepared via thermal CVD. The VACNT samples were placed on a button heater located in a stainless steel, cold wall reaction chamber (Figure S1b, Supporting Information). Once the temperature of the VACNTs reached $600{ }^{\circ} \mathrm{C}$, disilane, as the sole precursor, was introduced into the chamber over a period of $30 \mathrm{~min}$. During the silicon deposition, the chamber pressure was maintained at $\sim 1$ Torr. The as-grown VACNT-Si composite samples were then placed into a tube furnace and annealed at $800{ }^{\circ} \mathrm{C}$ for $30 \mathrm{~min}$ in argon (Ar) to improve the crystallinity of the $\mathrm{Si}$ coating in the VACNT-Si composites.

Fabrication of VACNT-Si (or GaN) noninfiltrated composite structures: Noninfiltrated VACNT-Si composite structures were fabricated via laser-assisted CVD (Figure S1c, Supporting Information). The VACNT samples were placed in a stainless steel, cold wall CVD chamber. A high power carbon dioxide $\left(\mathrm{CO}_{2}\right)$ laser was used for the heating source and directly irradiated the backside of the VACNTs. When the temperature of the VACNTs became stable at $600{ }^{\circ} \mathrm{C}$, under laser irradiation, disilane was introduced as the sole precursor over a period of $5 \mathrm{~min}$. For 
comparison, VACNT-GaN composites were also fabricated using the same laser-assisted CVD system. The experimental parameters used for the GaN growth were similar to those reported by Golgir et al. ${ }^{35}$ In brief, trimethylgallium (TMGa) and ammonia $\left(\mathrm{NH}_{3}\right)$ were used as the precursors. The growth temperature and deposition time were $900{ }^{\circ} \mathrm{C}$ and $1 \mathrm{~min}$, respectively. During the $\mathrm{Si}$ and $\mathrm{GaN}$ deposition process, the chamber pressure was maintained at $\sim 1$ Torr and $\sim 100$ Torr, respectively.

High-temperature antioxidative ability and thermal stability test: For antioxidative ability testing, the as-prepared bulk and cubic VACNT/ceramic ( $\mathrm{Si}$ or $\mathrm{GaN}$ ) composite structures were oxidized in a muffle furnace (Thermolyne ${ }^{\mathrm{TM}}$ Benchtop $1100^{\circ} \mathrm{C}$ ) at different temperatures until they failed. The thermal stability test was carried out in a tube furnace (MTI Corporation, GSL-1500XRTP50) with temperatures ranging from 600 to $1400{ }^{\circ} \mathrm{C}$ in vacuum ( 10 mTorr).

Temperature-dependent electrical conductivity measurement: The electrical properties of as-prepared samples were measured using a homemade electrical conductivity measurement system. The measurement system consisted of a semiconductor parameter analyzer (Agilent 4155C), a heating chamber (Linkam Scientific Instruments (UK), 77-800 K, THMS600), a heating power/temperature controller, and a probe station (Cascade Microtech, MPS 150). A cooling water system was used when the measurement temperature was higher than $573 \mathrm{~K}$. A liquid nitrogen tank was used for providing a nitrogen environment. Before the test, VACNTs and VACNT-Si composite devices were loaded into the chamber and placed at the center of the heating stage. A purging process was then performed to ensure all of the air was purged out. After that, the heating 
stage started to ramp up the temperature from 273 to $873 \mathrm{~K}$ at a rate of $10 \mathrm{~K} / \mathrm{min}$. An currentvoltage (I-V) sweep was then performed at incremental temperature points of $25 \mathrm{~K}$.

Characterization: Room-temperature Raman spectral analysis was performed in a microRaman system (Renishaw, inVia ${ }^{\mathrm{TM}}$ Plus). The Raman scattering was excited by a $514 \mathrm{~nm} \mathrm{Ar}^{+}$laser with a power of $\sim 3 \mathrm{~mW}$. Raman spectra were collected through a 50x objective lens with an accumulation time of $10 \mathrm{~s}$ at each position. Atomic force microscopy ((AFM), Bruker, Multimode 8) operated in the peak force working mode was applied to determine the tube diameter of the asgrown VACNTs. Scanning electron microscopy ((SEM), Hitachi, S4700 Field-Emission SEM) was used to study the morphologies of the as-prepared VACNTs and VACNT-ceramic composites. 


\section{RESULTS AND DISCUSSION}

\section{Growth and characterization of VACNTs}

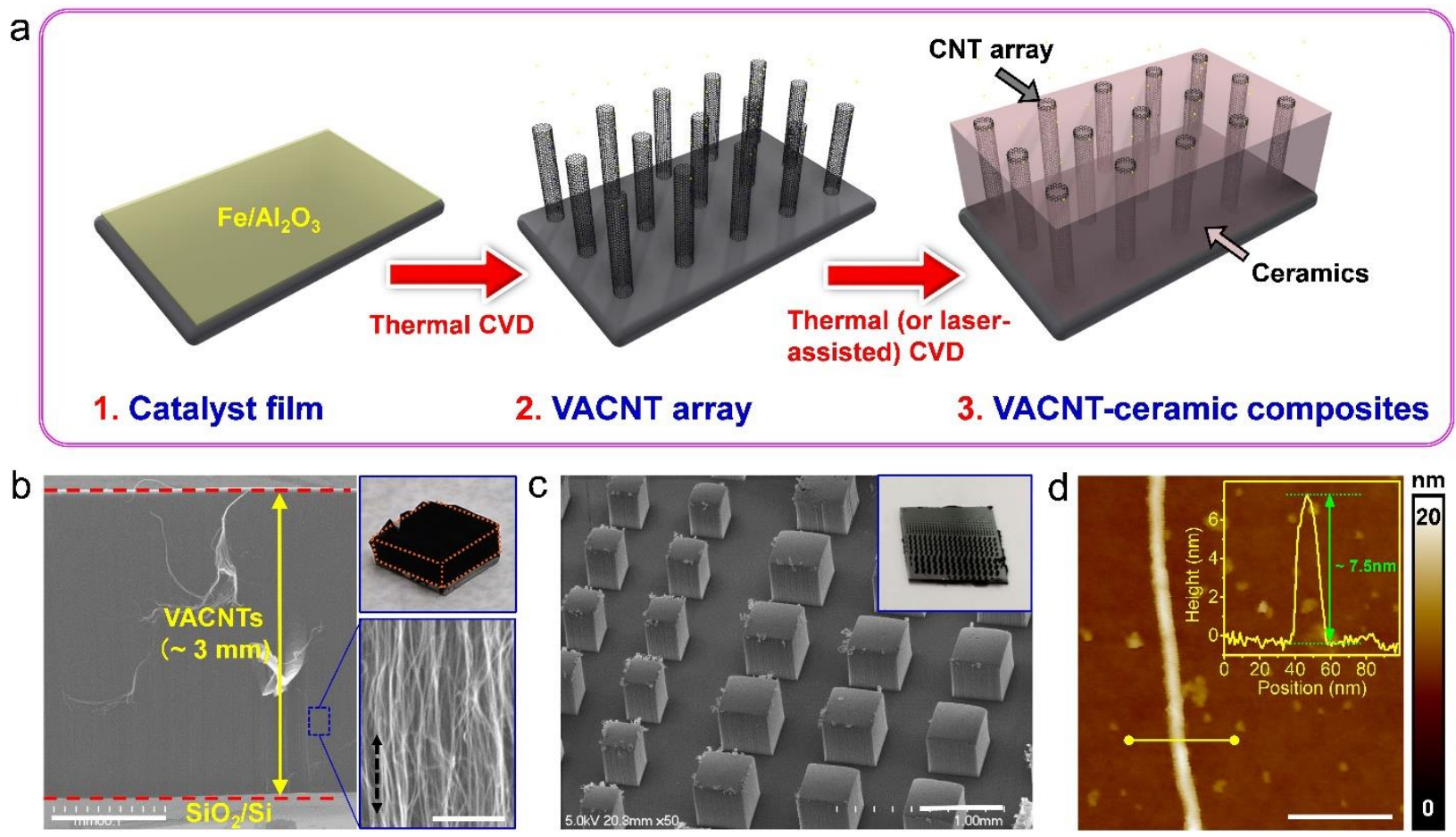

Figure 1. Growth and characterization of VACNTs. (a) A schematic of the fabrication process for VACNTSi composites. (b, c) SEM images of the as-grown (b) bulk and (c) patterned VACNTs using thermal CVD. Top insets in (b, c) show the corresponding photographs of actual samples, while the bottom inset in (b) displays an enlarged cross-sectional SEM image. (d) AFM topography of a typical dispersed single CNT. The inset in (d) shows the cross-sectional height profile along the yellow line. Scale bars: $1 \mathrm{~mm}$ for (b), $500 \mathrm{~nm}$ for the bottom inset of (b), $500 \mu \mathrm{m}$ for (c), and $100 \mathrm{~nm}$ for (d).

In this study, two types of VACNTs, used for the conducting network and building template, were prepared: ultralong bulk VACNTs $(\sim 3 \mathrm{~mm})$ (Figure $1 \mathrm{~b})$ and cubic patterned VACNTs (Figure 1c). As shown in Figure 1b, the as-grown VACNTs were dense and straight not only in the macroscale but also in the microscale (bottom inset in Figure 1b). Raman spectral analysis showed that the ratio of the D to the $\mathrm{G}$ band was about 1.1 (Figure S2a, Supporting Information), indicating a relatively high percentage of defects in the ultralong VACNTs. Figures S2b and 1d show the AFM 
topographies of the dispersed VACNT films and a typical single CNT, respectively. The average tube diameter was measured to be $\sim 7.5 \mathrm{~nm}$ (inset in Figure 1d), demonstrating multiwall carbon nanotubes (MWCNTs) produced using our two-zone thermal CVD method. Compared to singlewalled CNTs (SWCNTs), the MWCNTs exhibited enhanced electrical conductivity and thermal and chemical stabilities. ${ }^{36}$ Therefore, ultralong MWCNTs with large tube diameters were chosen as the templates for the fabrication of thermally stable and electrically conductive VACNT-ceramic composite structures.

\section{Fabrication of VACNT-ceramic (Si or GaN) composite structures}

Compared to the traditional high-temperature ceramics [such as $\mathrm{SiC}$ and boron nitride (BN)], both $\mathrm{Si}$ and GaN have good thermal stability, corresponding to a melting point of $\sim 1400$ and $\sim 2500{ }^{\circ} \mathrm{C}$, respectively. More importantly, both of them exhibit much higher electrical conductivity than that of traditional ceramics. Thus, Si $(\mathrm{GaN})$-based VACNT-ceramic composites will be fabricated, which are expected to show excellent electrical performance and have great potential as hightemperature electrode materials.

In general, thermal CVD heats the sample uniformly, which creates one-dimensional diffusion paths for reactants that are normal to the reaction plane. In contrast to the thermal CVD method, laser-assisted CVD heats locally and creates a sphere-shaped, three-dimensional (3D) diffusion path for reactants. Based on this difference, it is possible to realize controlled infiltrated and noninfiltrated composites using these two different growth methods: laser-assisted CVD for a noninfiltrated structure and thermal CVD for an infiltrated structure. 

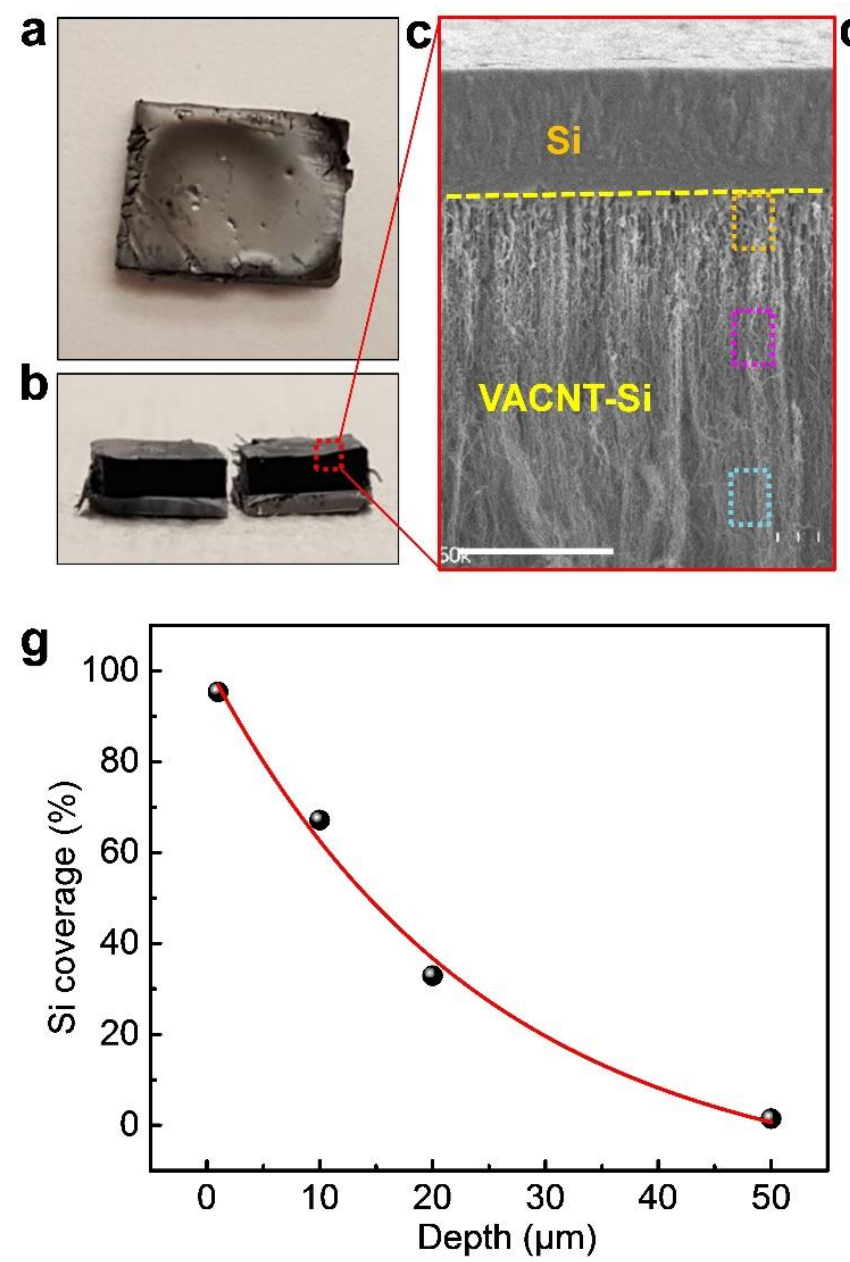
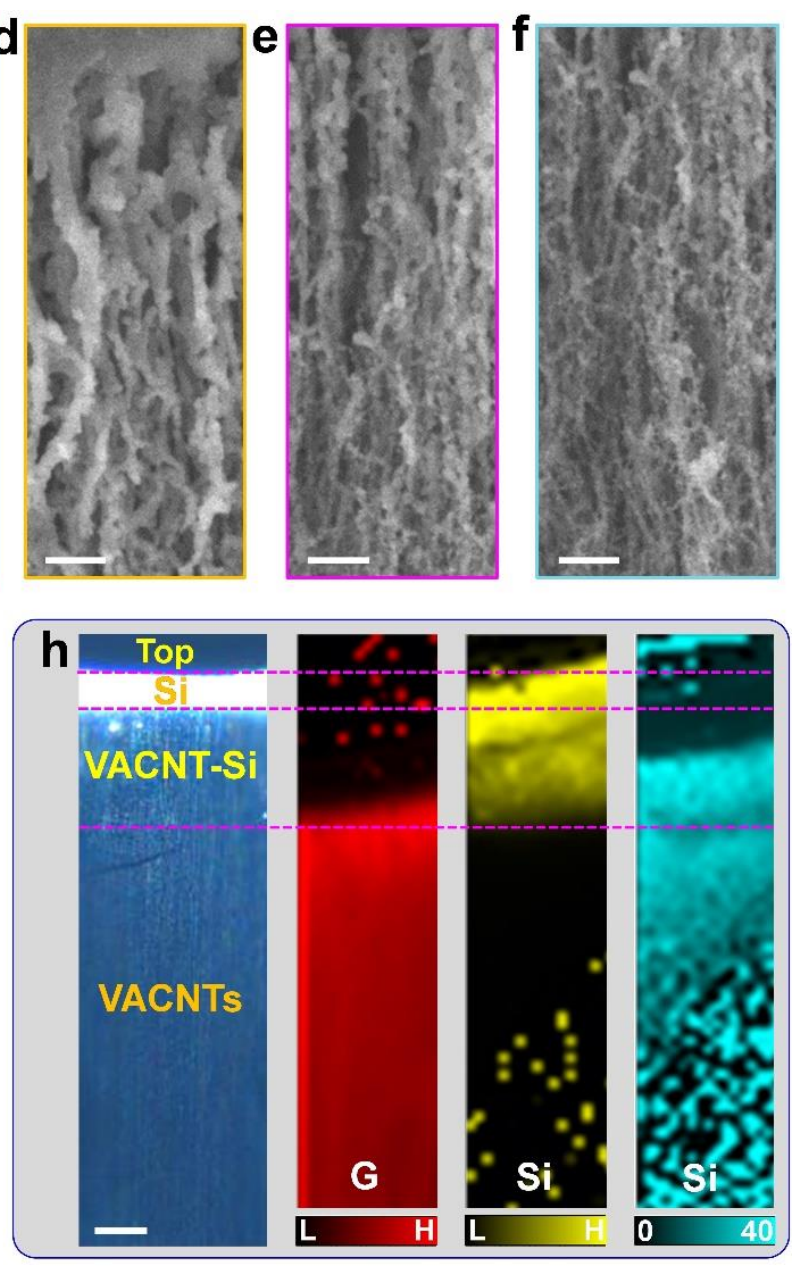

Figure 2. Fabrication of noninfiltrated VACNT-Si composite structures via laser-assisted CVD. (a, b) Optical images of as-prepared bulk VACNT-Si composites: (a) top view and (b) cross-sectional view. (c) Cross-sectional SEM image of the VACNT-Si composites. (d-f) Magnified SEM images shown in dashed squares in (c) from top to bottom. (g) Statistical results of the coverage of Si coating distributed on VACNTs from the top surface to the inside. (h) Raman mapping characterization of the cross-sectional area of a noninfiltrated VACNT-Si composite structure. From left to right in (h): optical image, G band $\left(\sim 1580 \mathrm{~cm}^{-1}\right)$ intensity mapping, Si peak $\left(\sim 520 \mathrm{~cm}^{-1}\right)$ intensity mapping, and full width at half maximum (FWHM) mapping for Si peak. Scale bars: $10 \mu \mathrm{m}$ for (c), $200 \mathrm{~nm}$ for (d-f), and $10 \mu \mathrm{m}$ for (h).

Figure 2a presents an optical image of the silicon deposited onto bulk VACNTs via laser-assisted CVD, forming noninfiltrated VACNT-Si composite structures. It can be seen that a layer of silvercolored silicon covered all of the VACNT top surfaces. In order to determine the infiltration status inside the VACNTs, the composite sample was carefully halved (Figure 2b). A silicon layer with a 
thickness of $\sim 10 \mu \mathrm{m}$ formed on top of the VACNTs under laser irradiation for $5 \mathrm{~min}$, corresponding to a fast growth rate of $\sim 2 \mu \mathrm{m} / \mathrm{min}$ (Figure $2 \mathrm{c}$ ). In addition, there was a significant structural change from the VACNT-Si top surface to the subsurface area (Figures 2d-f). In Figure 2d, a large amount of silicon is visible in the spacing between VACNTs at the top surface, forming fiber-like structures with much thicker tube diameters than those of the original VACNTs. At the slightly deeper areas (Figures 2e and 2f), quasi-continuous small silicon particles are attached to most of the CNT surfaces. Figure $2 \mathrm{~g}$ shows the statistical results of the coverage of $\mathrm{Si}$ coating distributed on VACNTs from the top surface to the inside, where no Si coating could be seen at a depth of $\sim 50$ $\mu \mathrm{m}$ from the VACNT top. To quantitatively evaluate the infiltration of silicon, cross-sectional Raman mapping was carried out (Figures $2 \mathrm{~h}$ and S3). The distribution of high-quality silicon tended to concentrate at the VACNT top surfaces. Low-quality silicon coating was deposited along the VACNTs at subsurfaces. There was no (strong) Raman signal for silicon (CNTs) beyond 50 $\mu \mathrm{m}$ in depth, indicating a poor infiltration of silicon inside the bulk VACNTs. Similar noninfiltrated structures were obtained for the VACNT-GaN composites using the same method (Figures S4 and S5, Supporting Information), which suggests that laser-assisted CVD is not suitable for fabricating infiltrated composite structures.

Surprisingly, VACNT-Si composite structures prepared by thermal CVD were totally different as compared to those prepared by laser-assisted CVD (Figure 3). The only difference between the two growth methods was that the heating source was changed from a $\mathrm{CO}_{2}$ laser (in LCVD) to a button heater (in thermal CVD). Figure 3a shows the cross-sectional SEM image of the as-prepared 
VACNT-Si composites in a thermal CVD system with the introduction of disilane at $600{ }^{\circ} \mathrm{C}$ for 30 min. No obvious thick layer of silicon coating could be identified on the VACNT top surfaces. As shown in the magnified SEM images in Figures 3b-d, the spacing among the VACNTs from the top surface to the bottom side were fully filled with deposited silicon, which indicates that infiltrated VACNT-Si composite structures were successfully fabricated via thermal CVD.
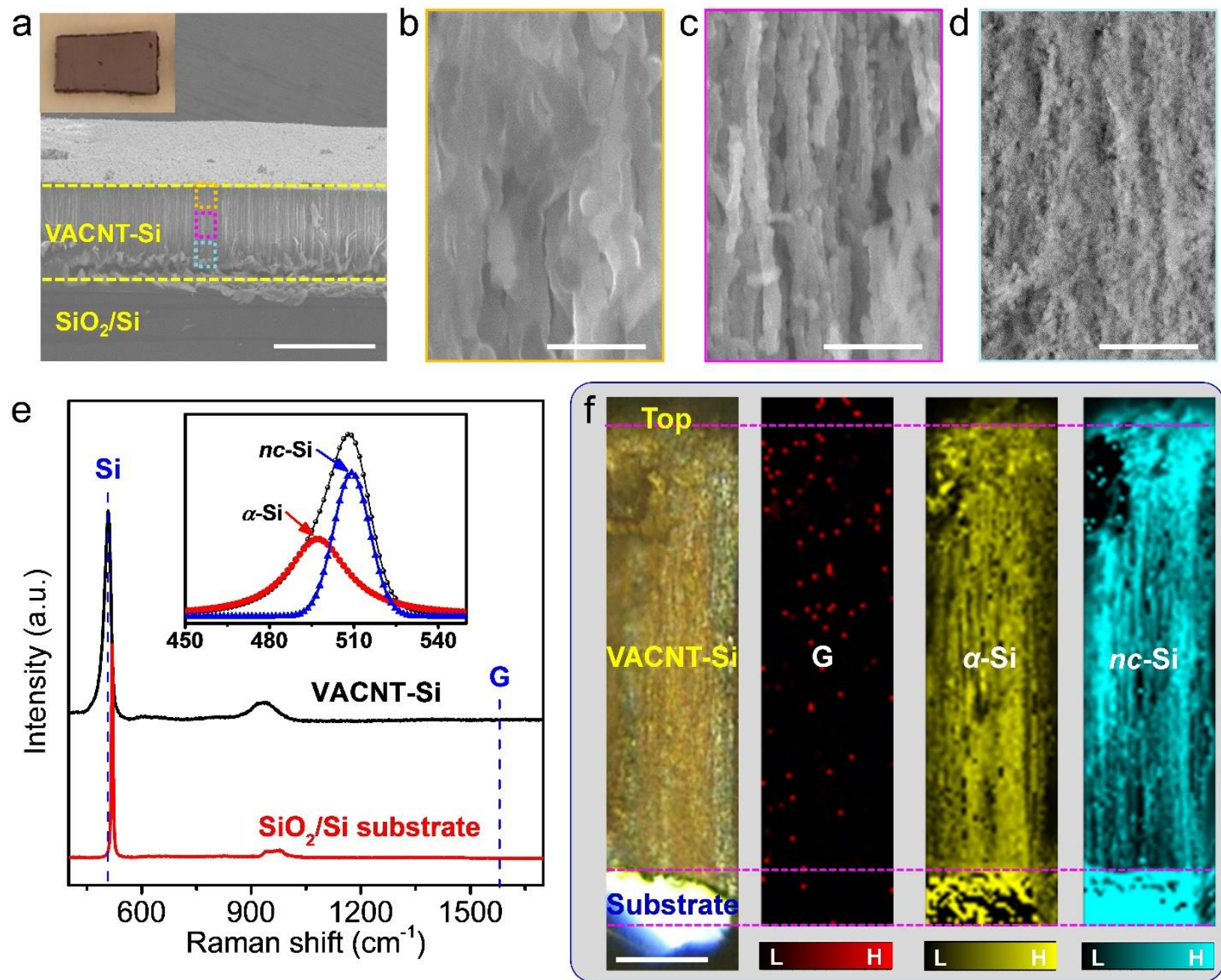

Figure 3. Fabrication of infiltrated VACNT-Si composite structures via thermal CVD. (a) Cross-sectional SEM image of VACNT-Si composites (Inset: top-view optical image). (b-d) Magnified SEM images shown in the dashed squares in (a) from top to bottom. (e) Typical Raman spectra for the VACNT-Si composites (black line) and $\mathrm{SiO}_{2} / \mathrm{Si}$ substrate (red line). Inset in (e) shows the Raman spectrum of the Si peak in VACNT-Si composites, where the black, red, and blue dotted lines correspond to the measured Raman 
spectrum and the Gaussian/Lorentzian mixed fittings for amorphous and monocrystalline phases, respectively. (f) Cross-sectional Raman mapping characterization of the infiltrated VACNT-Si composite structures. From left to right in (f): optical image, G-band intensity mapping at $\sim 1580 \mathrm{~cm}^{-1}$, a-Si intensity mapping at $\sim 497 \mathrm{~cm}^{-1}$, and $n c-S i$ intensity mapping at $\sim 509 \mathrm{~cm}^{-1}$. Scale bars: $200 \mu \mathrm{m}$ for (a), $500 \mathrm{~nm}$ for (b-d), and $30 \mu \mathrm{m}$ for (f).

Figure 3e shows a typical Raman spectrum for infiltrated VACNT-Si composite structures (black line). Only a Raman signal from the silicon (TO $520 \mathrm{~cm}^{-1}$ and $2 \mathrm{TO} 940 \sim 980 \mathrm{~cm}^{-1}$ ) was detected; no Raman signal of G-band from the VACNTs could be detected, which further revealed that VACNTs from the outside to the inside were fully covered by the silicon coating. Compared to the sharp Raman peak of silicon from the $\mathrm{SiO}_{2} / \mathrm{Si}$ substrate (red line in Figure 3e), the as-deposited silicon had a relatively broad Raman peak and could be well fitted by two different bands that originated from amorphous silicon $(\alpha-\mathrm{Si})$ at $\sim 497 \mathrm{~cm}^{-1}$ and nanocrystalline silicon $(n c-\mathrm{Si})$ at $\sim 509$ $\mathrm{cm}^{-1}$. We estimated the mean grain size $d$ of $n c$-Si in the VACNT-Si composites by employing the formula as follows, ${ }^{37}$

$$
d=2 \pi(\mathrm{B} / \Delta \omega)^{1 / 2}
$$

where $\Delta \omega$ is the peak shift of $n c$-Si related to the TO mode of single crystalline silicon at $520 \mathrm{~cm}^{-}$ ${ }^{1}$ and $\mathrm{B}$ is a constant value of 2.0 . From the peak position that $\Delta \omega \approx 11$, the mean grain size $d$ for $n c$-Si was caculated to be approximately $2.7 \mathrm{~nm}$. In additin, we deduced the volume fraction $X_{\mathrm{c}}$ of Si crystallinity in the VACNT-Si composites by using the equation, ${ }^{37}$

$$
X_{c}=I_{c} /\left(I_{c}+\eta I_{a}\right)
$$

where $I_{c}$ and $I_{a}$ are the Raman intensities for the $n c-\mathrm{Si}$ and $\alpha$-Si, and $\eta$ is a scattering factor 
which is about $1 .^{38}$ From Figure $3 \mathrm{e}$, the value of $X_{c}$ was calculated to be $\sim 70.6 \%$.

Raman mapping in Figure 3f shows that both $\alpha$-Si and $n c$-Si were distributed along the VACNTSi composites from the top surface to the bottom substrate. At the same time, no Raman signal from the G-band originated from VACNTs in the same regions, further indicating a full coverage of silicon over the VACNTs and a good uniformity of silicon infiltration across the composites.

Next, we discuss the growth mechanisms for infiltrated and noninfiltrated VACNT-Si composite structures via two different material deposition techniques: thermal CVD and laser-assisted CVD. In the case of thermal CVD, the temperature was uniformly distributed across all of the VACNTs. As a result, a chemical reaction occurred simultaneously inside and outside the VACNT arrays. Meanwhile, the diffusion path for precursors was typically one dimensional. ${ }^{39}$ On the contrary, the laser-assisted CVD method heated locally, which produced a temperature gradient across the sample and made the reaction process more complicated than that of thermal CVD. In our case, the heating zone in laser-assisted CVD was a hemisphere centered near the laser focal point, which created a 3D diffusion path for the precursors. As a result, the deposition rate of laser-assisted CVD was several orders of magnitude higher than that of thermal CVD. ${ }^{40}$ To better understand the formation of noninfiltrated VACNT-ceramic composite structures, we discuss the following two aspects in laser-assisted CVD: temperature distribution and gas diffusion.

Laser irradiation provides the main source of the thermal energy during the laser-assisted CVD process. In our case, silicon was directly deposited onto the VACNT arrays which possessed 
excellent thermal conductivity. ${ }^{41}$ Therefore, the thermal energy that was conducted away from the irradiation plane cannot be neglected. The conduction can be described by a general conduction equation for a given volume: ${ }^{39}$

$$
k\left(\frac{\partial^{2} T}{\partial x^{2}}+\frac{\partial^{2} T}{\partial y^{2}}+\frac{\partial^{2} T}{\partial z^{2}}\right)=k \nabla^{2} T=0
$$

Since the conductivity of VACNTs along the tube, $k$, was very high, the heat transfer along the VACNTs was significant, which caused the local temperature at the irradiation plane to be lower than expected. During the silicon deposition, the laser irradiated the $\mathrm{SiO}_{2} / \mathrm{Si}$ substrate beneath the VACNTs. The heat was then transferred to VACNTs for silicon deposition. In this case, the pathway for conduction was mainly along the direction of the nanotubes, which led to a massive heat transfer from the VACNTs/substrate interfaces to the tips of the VACNTs. In addition, the heat transfer between tubes was not favored in VACNTs, which restrained the heat inside them. ${ }^{39}$ Finally, silicon was deposited mainly on top surfaces of the VACNT arrays.

The CVD reaction rate is generally diffusion limited at high temperatures. ${ }^{39}$ In thermal CVD, reaction occurs simultaneously across all of the sample surfaces due to the uniform temperature distribution. During the material deposition, the gas diffusion was steadily dominated by the temperature which was beneficial for the infiltration of the reactants. However, laser-assisted CVD was slightly more complicated than thermal CVD. In laser-assisted CVD, the large temperature gradient across the whole sample can result in the temperature of some areas of the sample being not high enough. In these areas, the CVD reaction can be limited by chemical kinetics or by both 
chemical kinetics and diffusion. ${ }^{39}$ In addition, as the temperature near the laser focal point was very high, the deposition rate was highly limited by the reactants since the new reagents were not able to reach the reaction zone fast enough to feed the reaction. ${ }^{42}$ This explains the rapid formation of thick ceramics (silicon or $\mathrm{GaN}$ ) coatings on the surfaces of VACNTs via laser-assisted CVD. In summary, as a result of the significant difference in deposition rate caused by different temperature distribution and gas diffusion mechanisms, different composite structures could be obtained via these two methods.

\section{High-temperature antioxidative ability and thermal stability}

The as-prepared VACNTs showed good antioxidative ability at $\leq 550{ }^{\circ} \mathrm{C}$ but were massively oxidized at a temperature $\geq 600{ }^{\circ} \mathrm{C}$ (Figure S6, Supporting Information). This value is consistent with previous reports. ${ }^{43}$ Hence, we chose $600{ }^{\circ} \mathrm{C}$ as a starting oxidation test temperature to investigate the antioxidative ability of as-prepared, infiltrated and noninfiltrated VACNT-ceramic composite structures. The cubic VACNT-ceramic composite structures were prepared, as shown in Figure $4 \mathrm{a}$ (noninfiltrated VACNT-GaN composites) and Figure 4e (infiltrated VACNT-Si composites). To examine the existence of CNTs inside the VACNT-ceramic composites, some of the cubes were gently and precisely removed from the $\mathrm{SiO}_{2} / \mathrm{Si}$ substrates and transferred onto new substrates bottom up after the oxidation treatments. 

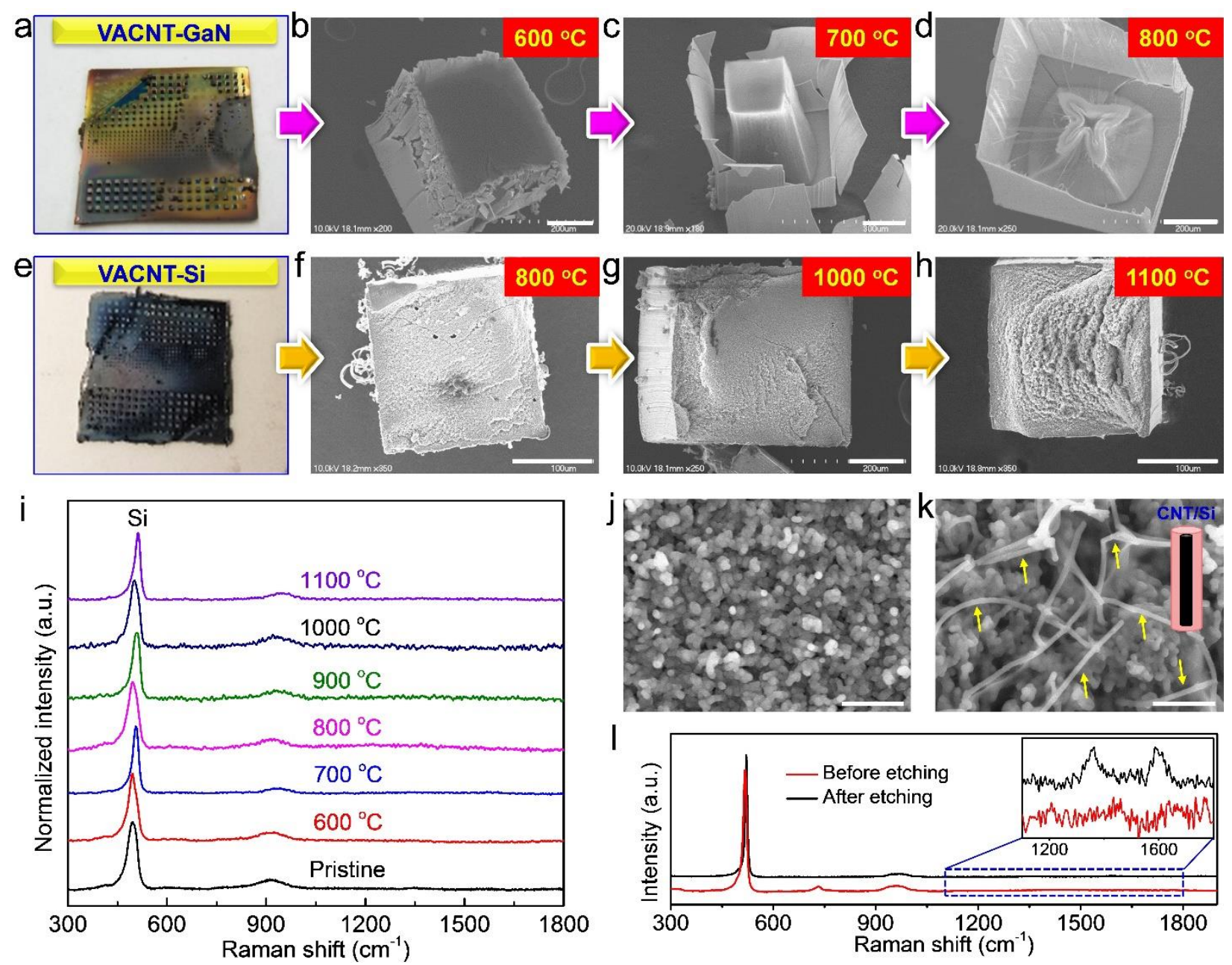

Figure 4. High-temperature antioxidative ability of the VACNT-ceramic composite structures. (a) Optical image of a patterned noninfiltrated VACNT-GaN composite sample prepared by laser-assisted CVD. (b-d) Typical SEM images of VACNT-GaN cubes after oxidation in air at (b) 600 , (c) 700, and (d) $800{ }^{\circ} \mathrm{C}$ for 5 min. (e) Optical image of an infiltrated VACNT-Si composite sample prepared by thermal CVD. (f-h) Typical SEM images of VACNT-Si cubes after oxidation in air at (f) 800 , (g) 1000 , and (h) $1100{ }^{\circ} \mathrm{C}$ for 5 min. (i) Raman spectra of VACNT-Si cubes after oxidation in air at different temperatures. (j, k) Top-view SEM images of oxidized infiltrated VACNT-Si composites (j) before and ( $k$ ) after etching treatments in 1 wt.\% sodium hydroxide $(\mathrm{NaOH})$ solution. (I) Typical Raman spectra for oxidized infiltrated VACNT-Si composites before (red line) and after (black line) etching treatments. Inset in (I) shows the magnified Raman spectra from 1100 to $1800 \mathrm{~cm}^{-1}$. Scale bars: $100 \mu \mathrm{m}$ for (b-d, f-h) and $2 \mu \mathrm{m}$ for $(\mathrm{j}, \mathrm{k})$.

Figures 4b-d compare the noninfiltrated VACNT-GaN composite structures after successive oxidation processing at 600,700 , and $800{ }^{\circ} \mathrm{C}$, respectively. It can be seen in Figure $4 \mathrm{~b}$ that VACNTs inside the GaN coatings remained intact after oxidation at $600{ }^{\circ} \mathrm{C}$, suggesting a better antioxidative 
ability as compared to bare VACNTs. After oxidation at $700{ }^{\circ} \mathrm{C}$ for 5 min (Figure $4 \mathrm{c}$ ), VACNTs inside the GaN coatings ended up in a trapezoid-shaped structure. In addition, the top surfaces were considerably smaller than the bottom surfaces, revealing a sign of oxidation. VACNTs inside the GaN coatings were fully oxidized after a further oxidation treatment at $800{ }^{\circ} \mathrm{C}$ (Figure $4 \mathrm{~d}$ ). Although it is difficult to realize in situ real-time monitoring of the high-temperature oxidation process, the observed shape change in VACNT-GaN composites revealed a potential oxygen leak through the thin GaN coatings $(\sim 1 \mu \mathrm{m})$. In particular, the oxygen leak happened mostly from the bottom edge and partially from the sidewall of the GaN coatings. It has been reported that laserassisted CVD leads to an ultrafast growth of $\mathrm{GaN} .{ }^{35}$ As a result, lots of stresses are embedded inside GaN coating, which may cause nano-/microcracks during the GaN growth. This explains why noninfiltrated VACNT-GaN composite structures prepared by laser-assisted CVD demonstrate a slight improvement in antioxidative ability compared to bare VACNTs.

On the other hand, infiltrated VACNT-Si composite structures demonstrated excellent antioxidative capability (Figures 4f-h). The SEM image in Figure 4f shows an upside-down view of one typical cube of VACNT-Si composite after oxidation at $800{ }^{\circ} \mathrm{C}$ for $5 \mathrm{~min}$. No obvious change in the shape or loss of CNTs was observed in the VACNT-Si composites. After processing at higher oxidation temperatures, ranging from 1000 to $1100{ }^{\circ} \mathrm{C}$ (Figures $4 \mathrm{~g}$ and $4 \mathrm{~h}$ ), infiltrated VACNT-Si cube structures retained their original shapes, indicating no obvious oxidation, and, namely, showed excellent high-temperature antioxidative capability. To confirm this result, Raman spectra of the sample after oxidation in air at different temperatures were also measured (Figure 4i), where 
only two Raman features of TO mode at $\sim 510 \mathrm{~cm}^{-1}$ and $2 \mathrm{TO}$ mode at $\sim 900 \mathrm{~cm}^{-1}$ that originated from Si were observed, ${ }^{44}$ while no D-band or G-band from VACNTs could be detected, suggesting a good overall coverage of Si around the CNT sample surfaces. A slight change in volume fraction $X_{\mathrm{c}}$ and no significant change in Si peak intensity/position during the process of oxidation indicate good high-temperature antioxidation ability of infiltrated VACNT-Si composites.

To further confirm CNTs were still present in the VACNT-Si composite structures after ultrahightemperature oxidation at $1100{ }^{\circ} \mathrm{C}$, a $\mathrm{NaOH}$ etching treatment was carried out to remove the silicon coating. The etch rate of the silicon was controlled to be as low as possible to ensure that only a thin layer of silicon (or silicon oxide) coating on the top surface was etched away. Figures $4 \mathrm{j}$ and 4k show top view SEM images of the same sample before and after etching the silicon coatings, respectively. No bare CNTs can be seen before etching (Figure 4j), while CNT-like structures with large tube diameters $(\sim 200 \mathrm{~nm})$, possibly composed of core/shell CNT-Si hybrids, could be observed after the etching process (Figures $4 \mathrm{k}$ and S7b, Supporting Information). In addition, we also found some CNT nanostructures along the edge of the composites (Figures S7c and S7d, Supporting Information), providing direct evidence of the existence of CNTs in VACNT-Si composite structures after ultrahigh-temperature oxidation. Figure 41 compares the Raman spectra of the oxidized VACNT-Si composites with and without etching treatments. Before etching treatment, only a sharp Raman peak at $\sim 520 \mathrm{~cm}^{-1}$ originating from the silicon coating was observed; while after the etching treatment, weak Raman signals of both the D-band and G-band appeared (inset in Figure 41), further demonstrating that CNTs still existed in the infiltrated VACNT-Si 
composites after high-temperature oxidation treatment.

We then investigated the thermal stability of infiltrated VACNT-Si composite structures. Infiltrated VACNT-Si composites were heated in vacuum from 600 to $1400^{\circ} \mathrm{C}$ for $30 \mathrm{~min}$ at each temperature (Figure S8, Supporting Information). The VACNT-Si composites showed a color change from gray to dark green at $\sim 1200{ }^{\circ} \mathrm{C}$ due to a change in surface morphology or chemical components. Raman spectra in Figure S8b show that the silicon signal vanished and a carbon signal appeared at $\sim 1200^{\circ} \mathrm{C}$, which indicates a possible phase change of VACNT-Si composites at this temperature point. After a further annealing at $1400{ }^{\circ} \mathrm{C}$, an evident $\mathrm{SiC}$ Raman peak at $\sim 790 \mathrm{~cm}^{-1}$ emerged, which suggests that CNTs reacted with the silicon coating at the VACNT-Si interfaces. As shown in Figures S8c and S8d, CNT-Si (or SiC) core-shell nanorods with hexagonal-shaped tips can be seen after annealing at $1400{ }^{\circ} \mathrm{C}$. We attributed this to the recrystallization of silicon during the phase change at a temperature around the melting point. Although the infiltrated VACNT-Si composites could not withstand $1400{ }^{\circ} \mathrm{C}$ due to rapid heating, they may have been transformed into VACNT-SiC composites under a mild heating process and obtained new functionalities.

\section{High velocity gas erosion resistance}

In this section, we discuss our investigation of high velocity gas erosion resistance of the patterned infiltrated VACNT-Si composites. Figure 5a shows the schematic of the infiltrated VACNT-Si composites placed under an oxy-acetylene combustion flame for high-velocity, high-temperature processing, where a high-velocity, hot oxy-fuel gas mixture was kept flowing across the sample surfaces. Figures $5 \mathrm{~b}$ and $5 \mathrm{c}$ show the top view SEM images of a cube of infiltrated VACNT-Si 
composite structure before processing. Raman spectrum in the inset of Figure $5 \mathrm{c}$ suggests the pristine status of the VACNT-Si sample. After the high velocity oxy-fuel flame treatment (Figure 5d), one can see that the shape of the cube structure was slightly deformed due to the high velocity, hot gas erosion, since an oxy-acetylene combustion flame can provide not only high temperature but also generate high flame velocity. In addition, the height of the cube was slightly decreased, which suggests that part of the CNTs in the composites were oxidized. Raman spectrum in the inset of Figure 5e shows that the silicon signal at $\sim 510 \mathrm{~cm}^{-1}$ became very weak and a new Raman peak appeared at $\sim 792 \mathrm{~cm}^{-1}$ that originated from the $\mathrm{SiC}$, a change considered to be induced by the reaction between VACNTs and silicon coatings during the ultrahigh-temperature oxy-fuel flame processing. ${ }^{45}$ In addition, carbon species also emerged after the flame processing, as evidenced by the appearance of a strong D-band $\left(1350 \mathrm{~cm}^{-1}\right)$ and a $\mathrm{G}$ band $\left(1613 \mathrm{~cm}^{-1}\right)$. However, the origin of the carbon signal is still unknown. It either originated from the VACNTs or the deposited amorphous carbon in the flame.
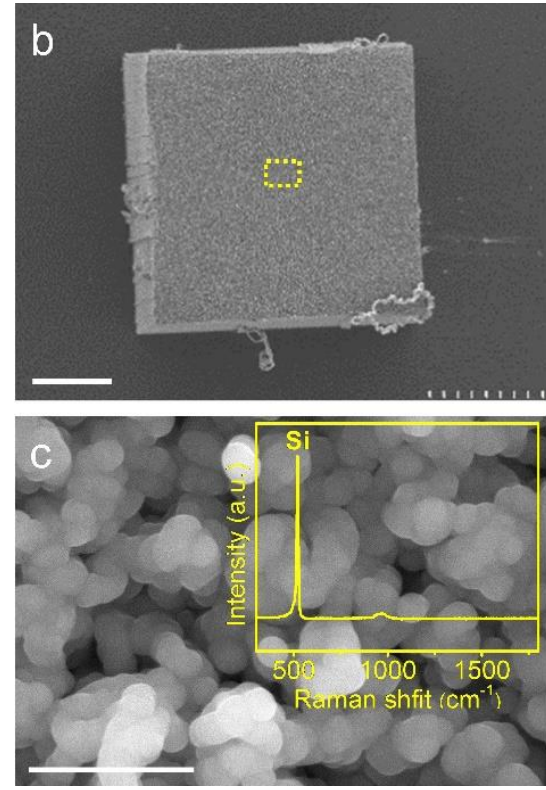
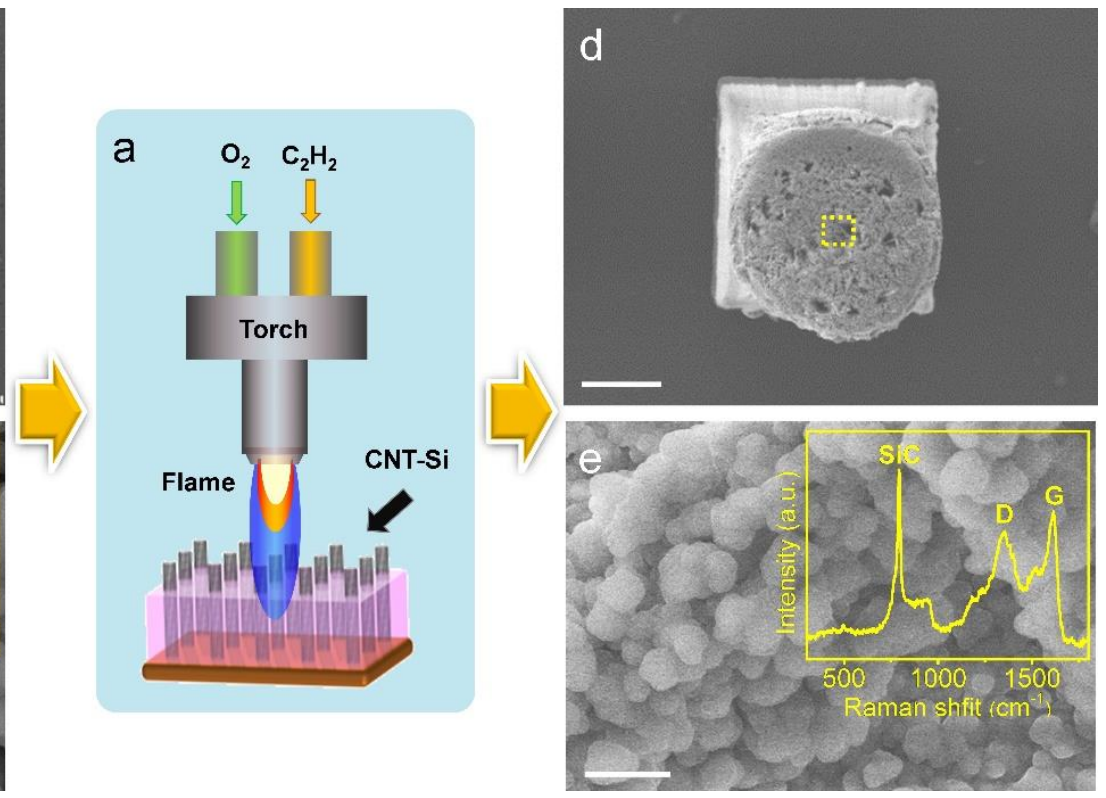
Figure 5. High velocity gas erosion resistance characterization of the infiltrated VACNT-Si composite structures using oxy-acetylene combustion flames. (a) A schematic experimental setup of the oxy-acetylene combustion flame. (b, d) Low and (c, e) high magnification SEM images of the infiltrated VACNT-Si cubic samples (b, c) before and ( $d, e)$ after processing via high velocity, high-temperature oxy-fuel flames. Insets in (c, e) show the corresponding Raman spectra. Scale bars: $50 \mu \mathrm{m}$ for (b, d) and $500 \mathrm{~nm}$ for (c, e).

\section{Electrical conductivity}

The room temperature $I-V$ characteristics of VACNTs and VACNT-Si composites are shown in Figures $6 \mathrm{a}$ and $6 \mathrm{~b}$, respectively. It can be seen that VACNTs exhibited an excellent linear $I-V$ characteristic with a bulk electrical resistance (conductivity) of $\sim 72 \Omega\left(\sim 171.36 \mathrm{Sm}^{-1}\right)$. It should be noted that the measured electrical conductivity of our grown VACNTs was much lower compared to previously reported results, ${ }^{46}$ which was probably caused by a high contact resistance between the copper substrate and the VACNTs. The infiltrated VACNT-Si composite device also exhibited a linear $I-V$ characteristic and had a relatively higher resistance (low conductivity) of $\sim 14.8 \mathrm{~K} \Omega\left(\sim 0.676 \mathrm{Sm}^{-1}\right)$, which was considered to be due to the silicon coating as the electrical contact covered the inside and outside of the VACNTs. Compared with the common CNT-ceramic conducting materials, the electrical conductivity of the as-prepared infiltrated VACNT-Si was lower than that of CNT-zirconium dioxide $\left(\mathrm{ZrO}_{2}\right)$ and CNT-aluminum oxide $\left(\mathrm{Al}_{2} \mathrm{O}_{3}\right)^{47,48}$ but higher than that of CNT-silicon nitride $\left(\mathrm{Si}_{3} \mathrm{~N}_{4}\right)$, to some extent. ${ }^{49}$ The room-temperature electrical conductivity of VACNT-Si composites after oxidation at different temperatures was also measured (Figure S9, Supporting Information). Compared to the VACNT-Si composites without oxidation, the electrical conductivity slightly decreased after being oxidized at $600{ }^{\circ} \mathrm{C}$, decreased by one magnitude after being oxidized at $700{ }^{\circ} \mathrm{C}$, and dropped significantly over $800{ }^{\circ} \mathrm{C}$. It is considered that, after oxidation treatment, the silicon on the VACNT-Si surface was oxidized but the oxidation 
is greatly slowed down beneath the VACNT-Si surface. ${ }^{50,51}$ The formed thin silicon oxide layer affected the measurements significantly by increasing the contact resistance between the sample and the electrode, which thus led to the decrease in the measured electrical conductivity.
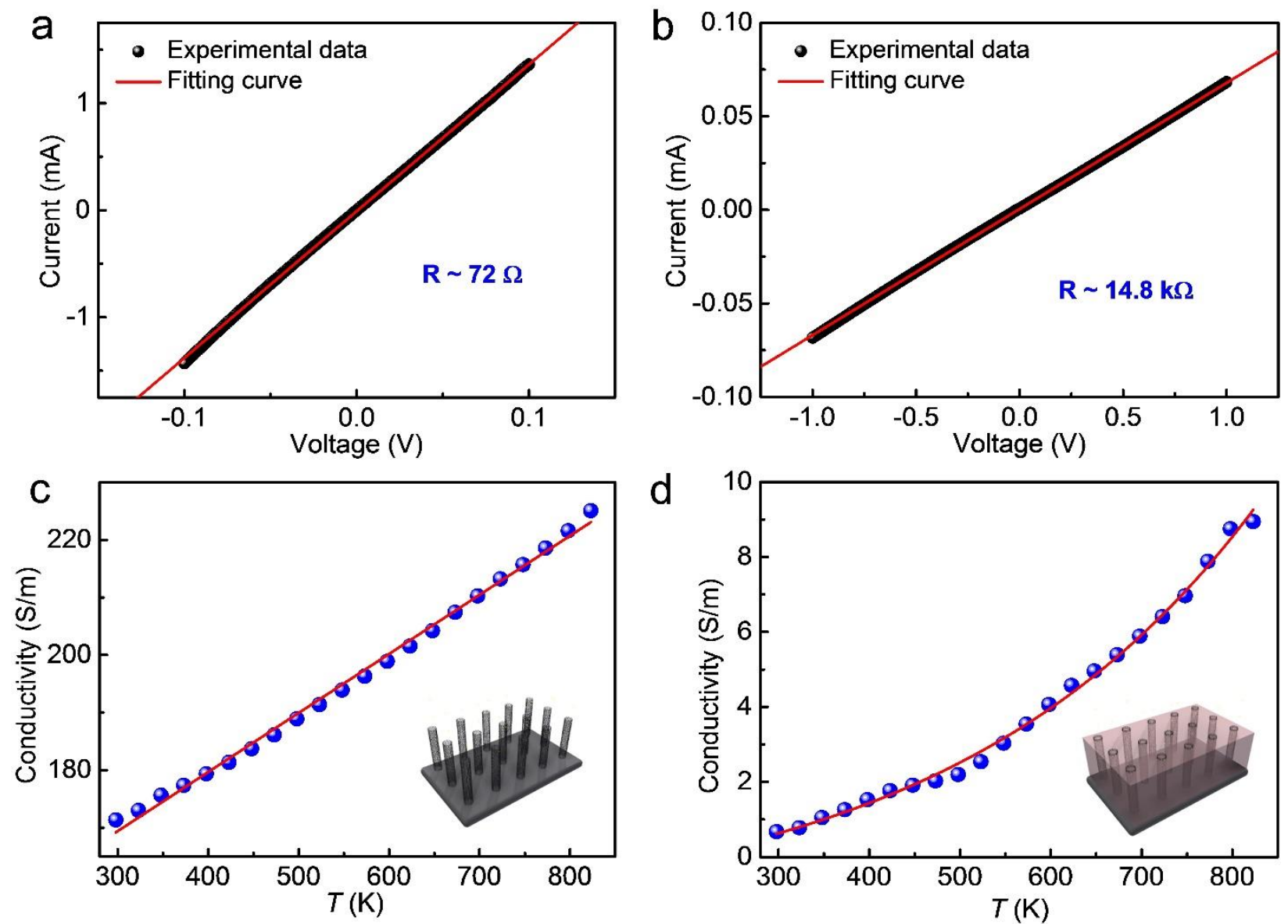

Figure 6. Comparative electrical conductivity of the infiltrated VACNT-Si composites and VACNTs. (a, b) Room temperature I-V curves of (a) VACNTs and (b) infiltrated VACNT-Si composites. (c, d) Temperaturedependent electrical conductivity of (a) VACNTs and (b) infiltrated VACNT-Si composites. Insets in (c, d) show schematics of the device structures.

We also investigated the temperature-dependent electrical conductivities of the VACNT-Si composites in comparison with those of VACNTs (Figure S10, Supporting Information). The electrical conductivity characteristics of both VACNTs and CNT-Si composites presented interesting changes as the temperature rose (Figures $6 \mathrm{c}$ and $6 \mathrm{~d}$ ). The electrical conductivity of the 
VACNTs increased linearly as the temperature increased, from $171.36 \mathrm{Sm}^{-1}$ (at $273 \mathrm{~K}$ ) to 225.08 $\mathrm{Sm}^{-1}$ (at $823 \mathrm{~K}$ ) (Figure 6c). The change in conductivity was less than a hundred $\mathrm{S} / \mathrm{m}$ with a rough increase of $31 \%$. In contrast, the electrical conductivity of the VACNT-Si composites showed an exponential increase as the temperature increased (Figure 6d), an indicator of the effect of ionic conducting. ${ }^{52}$ In addition, the electrical conductivity of infiltrated VACNT-Si composites increased significantly, from $0.676 \mathrm{Sm}^{-1}$ (at $273 \mathrm{~K}$ ) to $8.945 \mathrm{Sm}^{-1}$ (at $823 \mathrm{~K}$ ).

A qualified high-temperature electrode materials should be thermally stable, chemically inert, oxidation resistant, electrically conductive, and thermally conductive. In this work, we have fabricated VACNT-Si composite structures by controllably infiltrating Si into VACNT arrays, in which Si effectively serves as a shielding layer protecting the VACNTs from the oxidative and erosive environments, while the VACNTs mainly contribute to electrical conductivity. Comparing with some popular CNT-ceramic composites, such as $\mathrm{CNT}_{-} \mathrm{Al}_{2} \mathrm{O}_{3},{ }^{48,53} \mathrm{CNT}-\mathrm{MgO},{ }^{53}$ and CNT$\mathrm{Si}_{3} \mathrm{~N}_{4},{ }^{30}$ our fabricated VACNT-Si composite structures exhibit similar electrical conductivity and thermal/oxidation stability. In addition, considering the rapid increase in electrical conductivity at elevated temperature, it is therefore acceptable for the infiltrated CNT-Si composite structures to work as a promising hot electrode at high temperatures.

\section{CONCLUSIONS}

In summary, we have demonstrated a novel approach for the controlled fabrication of infiltrated and noninfiltrated VACNT-Si composite structures. Ultralong VACNTs $(\sim 3 \mathrm{~mm})$, as conducting networks and building framework templates, were successfully prepared, based on which 
uniformly infiltrated VACNT-Si composite structures were obtained via thermal CVD. Noninfiltrated VACNT-Si composite structures were produced via laser-assisted CVD. The asfabricated infiltrated VACNT-Si composites were proved to exhibit excellent thermal stability and good electrical conductivity and possess the ability to withstand various extreme conditions (e.g., high velocity hot gases), suggesting their great potential for high-temperature electrode applications. In addition, both thermal CVD and laser-assisted CVD proposed in this work require minimal complexity and effort in sample preparation, which is promising for the development of cost-effective, straightforward approaches to scalable high-temperature electrode material fabrication. In our further work, more efficient VACNT-based infiltrated ceramic composites (e.g., VACNT-BN) and their properties under extreme conditions will be explored.

\section{ASSOCIATED CONTENT}

Supporting Information. The Supporting Information is available free of charge on the ACS Publications website at http://pubs.acs.org.

(Figure S1) Schematics of the experimental setup for material growth; (Figure S2) Morphological and spectral characterization of the as-grown VACNTs; (Figure S3) Depth-dependent Raman spectra for the noninfiltrated VACNT-Si composites shown in Figure 2h; (Figures S4 and S5) Morphological and spectral characterization of noninfiltrated VACNT-GaN composite patterns prepared by laser-assisted CVD; (Figure S6) High-temperature antioxidative ability of VACNTs; (Figure S7) Morphological characterization of infiltrated VACNT-Si composites after etching treatment in $1 \mathrm{wt} . \% \mathrm{NaOH}$ solution; (Figure S8) Thermal stability characterization of the infiltrated 
VACNT-Si composite structures under ultrahigh temperature; (Figure S9) Electrical conductivity of the infiltrated VACNT-Si composites after oxidation in air at different temperatures; (Figure S10) Comparative electrical conductivity of the infiltrated VACNT-Si composites and VACNTs.

\author{
AUTHOR INFORMATION \\ * Corresponding Authors \\ E-mail: ylu2@unl.edu \\ E-mail: lidawei1008@gmail.com
}

NOTES

${ }^{ \pm}$Authors contributed equally.

\title{
ACKNOWLEDGMENTS
}

This research was financially supported by U.S. Department of Energy [NETL: DE-FE0023061, BES: DE-FOA-00001032], National Science Foundation (CMMI 1265122), and the Nebraska Center for Energy Science Research. 


\section{References}

(1) Jiang, S. P.; Liu, L.; Ong, K. P.; Wu, P.; Li, J.; Pu, J. Electrical Conductivity and Performance of Doped LaCrO3 Perovskite Oxides for Solid Oxide Fuel Cells. J. Power Sources 2008, $176(1), 82-89$.

(2) Rohatgi, V. K. High Temperature Materials for Magnetohydrodynamic Channels. Bull. Mater. Sci. 1984, 6 (1), 71-82.

(3) Whittingham, M. S.; Huggins, R. A. Measurement of Sodium Ion Transport in Beta Alumina Using Reversible Solid Electrodes. J. Chem. Phys. 1971, 54 (1), 414-416.

(4) Zhang, G. J.; Yan, Z.; Liu, Y. S.; Yasuoka, K.; Ishii, S. Influence of Electrode Contact on Luminescence from Alumina Ceramic Surface under Ac Electric Field in Vacuum. Appl. Phys. Lett. 2001, 78 (5), 625-627.

(5) Chamberlain, A. L.; Fahrenholtz, W. G.; Hilmas, G. E.; Ellerby, D. T. High-Strength Zirconium Diboride-Based Ceramics. J. Am. Ceram. Soc. 2004, 87 (6), 1170-1172.

(6) Birkby, I.; Stevens, R. Applications of Zirconia Ceramics. Key Eng. Mater. 1996, 122$124,527-552$.

(7) Fonseca, F. C.; Muccillo, R. Impedance Spectroscopy of (Yttria-Stabilized Zirconia)Magnesia Ceramic Composites. Solid State Ionics 2000, 131 (3-4), 301-309.

(8) Hibino, M.; Abe, K.; Mochizuki, M.; Miyayama, M. Amorphous Titanium Oxide Electrode for High-Rate Discharge and Charge. J. Power Sources 2004, 126 (1-2), 139143.

(9) Barbe, C. J. C.; Arendse, F.; Comte, P.; Jirousek, M.; Lenzmann, F.; Shklover, V.; Gra, 
M.; Gratzel, M. Nanocrystalline Titanium Oxide Electrodes for Photovoltaic Applications.

J. Am. Ceram. Soc. 1997, 80 (12), 3157-3171.

(10) Damm, D. L.; Fedorov, A. G. Radiation Heat Transfer in SOFC Materials and

Components. J. Power Sources 2005, 143 (1-2), 158-165.

(11) Singhal, S. C.; Wachsman, E. D. High Temperature Ion Conducting Ceramics.

Electrochem. Soc. Interface 2001, 10 (2), 22-27.

(12) Richter, D.; Sakharov, S.; Forsen, E.; Mayer, E.; Reindl, L.; Fritze, H. Thin Film

Electrodes for High Temperature Surface Acoustic Wave Devices. Procedia Eng. 2011, $25,168-171$.

(13) Ciftyürek, E.; Sabolsky, K.; Sabolsky, E. M. Platinum Thin Film Electrodes for HighTemperature Chemical Sensor Applications. Sensors Actuators, B Chem. 2013, 181, 702714.

(14) Cui, L.; Yang, Y.; Hsu, C.; Cui, Y. Carbon - Silicon Core - Shell Nanowires as High Capacity Electrode for Lithium Ion Batteries. Nano Lett. 2009, 9 (9), 3370-3374.

(15) Thackeray, M. M.; Kang, S.-H.; Johnson, C. S.; Vaughey, J. T.; Benedek, R.; Hackney, S. A. Li2MnO3-Stabilized LiMO2 (M = Mn, Ni, Co) Electrodes for Lithium-Ion Batteries. J. Mater. Chem. 2007, 17 (30), 3112-3125.

(16) Jeong, G.; Kim, M.; Han, J.; Kim, H. J.; Shul, Y. G.; Cho, E. High-Performance Membrane-Electrode Assembly with an Optimal Polytetrafluoroethylene Content for High-Temperature Polymer Electrolyte Membrane Fuel Cells. J. Power Sources 2016, $323,142-146$. 
(17) Picot, O. T.; Rocha, V. G.; Ferraro, C.; Ni, N.; D’Elia, E.; Meille, S.; Chevalier, J.;

Saunders, T.; Peijs, T.; Reece, M. J.; Saiz, E. Using Graphene Networks to Build

Bioinspired Self-Monitoring Ceramics. Nat. Commun. 2017, 8, 14425.

(18) Zhang, Q.; Lin, D.; Deng, B.; Xu, X.; Nian, Q.; Jin, S.; Leedy, K. D.; Li, H.; Cheng, G. J.

Flyweight, Superelastic, Electrically Conductive, and Flame-Retardant 3D Multi-

Nanolayer Graphene/Ceramic Metamaterial. Adv. Mater. 2017, 1605506.

(19) Samal, S. S.; Bal, S. Carbon Nanotube Reinforced Ceramic Matrix Composites- A

Review. J. Miner. Mater. Charact. Eng. 2008, 7 (4), 355-370.

(20) Curtin, W. A.; Sheldon, B. W. CNT-Reinforced Ceramics and Metals. Mater. Today 2004, $7(11), 44-49$.

(21) Popov, V. N. Carbon Nanotubes: Properties and Application. Mater. Sci. Eng. R Reports 2004, 43 (3), 61-102.

(22) Wang, Y.; Voronin, G. A.; Zerda, T. W.; Winiarski, A. SiC-CNT Nanocomposites: High Pressure Reaction Synthesis and Characterization. J. Phys. Condens. Matter 2006, 18 (1), 275-282.

(23) Shim, W.; Kwon, Y.; Jeon, S.-Y.; Yu, W.-R. Optimally Conductive Networks in Randomly Dispersed CNT:graphene Hybrids. Sci. Rep. 2015, 5, 16568.

(24) Zhang, T.; Kumari, L.; Du, G. H.; Li, W. Z.; Wang, Q. W.; Balani, K.; Agarwal, A. Mechanical Properties of Carbon Nanotube-Alumina Nanocomposites Synthesized by Chemical Vapor Deposition and Spark Plasma Sintering. Compos. Part A Appl. Sci. Manuf. 2009, 40 (1), 86-93. 
(25) Cho, J.; Boccaccini, A. R.; Shaffer, M. S. P. Ceramic Matrix Composites Containing Carbon Nanotubes. J. Mater. Sci. 2009, 44 (8), 1934-1951.

(26) Mahjouri-Samani, M.; Zhou, Y. S.; Fan, L.; Gao, Y.; Xiong, W.; More, K. L.; Jiang, L.; Lu, Y. F. Laser-Assisted Solid-State Synthesis of Carbon Nanotube/silicon Core/shell Structures. Nanotechnology 2013, 24 (25), 255604.

(27) Higgins, D. C.; Choi, J.-Y.; Wu, J.; Lopez, A.; Chen, Z. Titanium Nitride-carbon Nanotube Core-shell Composites as Effective Electrocatalyst Supports for Low Temperature Fuel Cells. J. Mater. Chem. 2012, 22 (9), 3727-3732.

(28) Balázsi, C.; Shen, Z.; Kónya, Z.; Kasztovszky, Z.; Wéber, F.; Vértesy, Z.; Biró, L. P.; Kiricsi, I.; Arató, P. Processing of Carbon Nanotube Reinforced Silicon Nitride Composites by Spark Plasma Sintering. Compos. Sci. Technol. 2005, 65 (5), 727-733.

(29) Li, W. Z.; Wen, J. G.; Sennett, M.; Ren, Z. F. Clean Double-Walled Carbon Nanotubes Synthesized by CVD. Chem. Phys. Lett. 2003, 368 (3-4), 299-306.

(30) Balázsi, C.; Kónya, Z.; Wéber, F.; Biró, L. P.; Arató, P. Preparation and Characterization of Carbon Nanotube Reinforced Silicon Nitride Composites. Mater. Sci. Eng. C 2003, 23 (6-8), 1133-1137.

(31) Zhan, G.-D.; Kuntz, J. D.; Wan, J.; Mukherjee, A. K. Single-Wall Carbon Nanotubes as Attractive Toughening Agents in Alumina-Based Nanocomposites. Nat. Mater. 2003, 2 (1), 38-42.

(32) Stano, K. L.; Carroll, M.; Padbury, R.; McCord, M.; Jur, J. S.; Bradford, P. D. Conformal Atomic Layer Deposition of Alumina on Millimeter Tall, Vertically-Aligned Carbon 
Nanotube Arrays. ACS Appl. Mater. Interfaces 2014, 6 (21), 19135-19143.

(33) Hao, M.; Kumar, A.; Hodson, S. L.; Zemlyanov, D.; He, P.; Fisher, T. S. Brazed Carbon Nanotube Arrays: Decoupling Thermal Conductance and Mechanical Rigidity. $A d v$.

Mater. Interfaces 2017, 4 (5), 1601042.

(34) Wu, T. C.; Chang, S. H. Temperature Enhanced Growth of Ultralong Multi-Walled Carbon Nanotubes Forest. Curr. Appl. Phys. 2009, 9 (5), 1117-1121.

(35) Golgir, H. R.; Gao, Y.; Zhou, Y. S.; Fan, L.; Thirugnanam, P.; Keramatnejad, K.; Jiang, L.; Lu, Y. F. Low-Temperature Growth of Crystalline Gallium Nitride Films Using Vibrational Excitation of Ammonia Molecules in Laser-Assisted Metalorganic Chemical Vapor Deposition. Cryst. Growth Des. 2014, 14 (12), 6248-6253.

Han, Z.; Fina, A. Thermal Conductivity of Carbon Nanotubes and Their Polymer Nanocomposites: A Review. Prog. Polym. Sci. 2011, 36 (7), 914-944.

He, Y.; Yin, C.; Cheng, G.; Wang, L.; Liu, X.; Hu, G. Y. The Structure and Properties of Nanosize Crystalline Silicon Films. J. Appl. Phys. 1994, 75 (2), 797-803.

Beeman, D.; Tsu, R.; Thorpe, M. F. Structural Information from the Raman Spectrum of Amorphous Silicon. Phys. Rev. B 1985, 32 (2), 874-878.

(39) Duty, C.; Jean, D.; Lackey, W. J. Laser Chemical Vapour Deposition: Materials, Modelling, and Process Control. Int. Mater. Rev. 2001, 46 (6), 271-287.

(40) D.Bauerle. Laser Processing and Diagnostics. In Springer Series in Chemical Phsics 39; $1983 ; 166-182$.

(41) Qiu, L.; Wang, X.; Su, G.; Tang, D.; Zheng, X.; Zhu, J.; Al., E. Remarkably Enhanced 
Thermal Transport Based on a Flexible Horizontally-Aligned Carbon Nanotube Array Film. Sci. Rep. 2016, 6, 21014.

(42) Allen, S. D.; Tringubo, A. B. Laser Chemical Vapor Deposition of Selected Area Fe and W Films. J. Appl. Phys. 1983, 54 (3), 1641-1643.

(43) Frank, B.; Rinaldi, A.; Blume, R.; Schlögl, R.; Su, D. S. Oxidation Stability of Multiwalled Carbon Nanotubes for Catalytic Applications. Chem. Mater. 2010, 22 (15), $4462-4470$.

(44) Galeener, F. L.; Lucovsky, G. Longitudinal Optical Vibrations in Glasses: $\mathrm{GeO}_{2}$ and $\mathrm{SiO}_{2}$. Phys. Rev. Lett. 1976, 37 (22), 1474-1478.

(45) Gorthy, P.; G., M. P. Production of Silicon Carbide from Rice Husks. J. Am. Ceram. Soc. 2004, 82 (6), 1393-1400.

(46) Wu, M. Le; Chen, Y.; Zhang, L.; Zhan, H.; Qiang, L.; Wang, J. N. High-Performance Carbon Nanotube/Polymer Composite Fiber from Layer-by-Layer Deposition. ACS Appl. Mater. Interfaces 2016, 8 (12), 8137-8144.

(47) Ahmad, I.; Yazdani, B.; Zhu, Y. Recent Advances on Carbon Nanotubes and Graphene Reinforced Ceramics Nanocomposites. Nanomaterials 2015, 5 (1), 90-114.

(48) Inam, F.; Yan, H.; Jayaseelan, D. D.; Peijs, T.; Reece, M. J. Electrically Conductive Alumina-Carbon Nanocomposites Prepared by Spark Plasma Sintering. J. Eur. Ceram. Soc. 2010, 30 (2), 153-157.

(49) Puchy, V. Tribological and Electrical Properties of Ceramic Matrix Composites with Carbon Nanotubes. Ceram. Int. 2012, 38, 5669-5676. 
(50) Mastail, C.; Bourennane, I.; Estève, a; Landa, G.; Rouhani, M. D.; Richard, N.;

Hémeryck, a. Oxidation of Germanium and Silicon Surfaces (100): A Comparative Study through DFT Methodology. IOP Conf. Ser. Mater. Sci. Eng. 2012, 41 (1), 12007.

(51) Klug, J.; Pérez, L. A.; Coronado, E. A.; Lacconi, G. I. Chemical and Electrochemical Oxidation of Silicon Surfaces Functionalized with APTES: The Role of Surface Roughness in the AuNPs Anchoring Kinetics. J. Phys. Chem. C 2013, 117 (21), 1131711327.

(52) Mason, T. O. Conductive Ceramics. Encyclopaedia Britannica; 2008.

(53) Flahaut, E.; Peigney, A.; Laurent, C.; Marlière, C.; Chastel, F.; Rousset, A. Carbon Nanotube-Metal-Oxide Nanocomposites: Microstructure, Electrical Conductivity and Mechanical Properties. Acta Mater. 2000, 48 (14), 3803-3812. 
Thermally Stable and Electrically Conductive, Vertically Aligned Carbon Nanotube/Silicon Infiltrated Composite Structures for High-Temperature Electrodes

Qi Ming Zou, Lei Min Deng, Da Wei Li, * Yun Shen Zhou, Hossein Rabiee Golgir, Kamran

Keramatnejad, Li Sha Fan, Lan Jiang, Jean-Francois Silvain, and Yong Feng Lu*

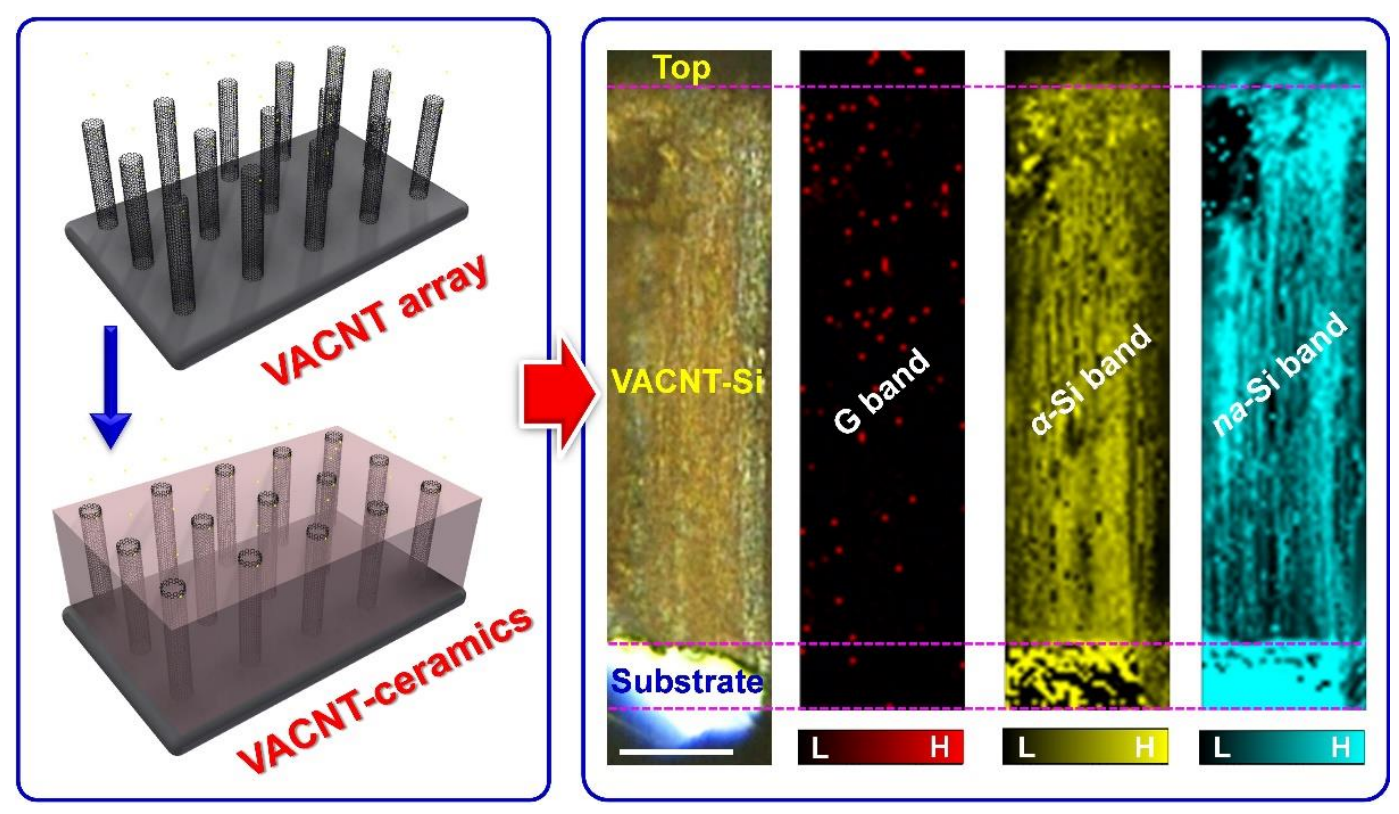

We have demonstrated the controllable fabrication of vertically aligned carbon nanotube-ceramic infiltrated composite structures via a single-step thermal chemical vapor deposition, and the structure exhibits excellent antioxidative ability (up to $1100{ }^{\circ} \mathrm{C}$ ), high thermal stability (up to $1400{ }^{\circ} \mathrm{C}$ ), good high velocity hot gas erosion resistance, and good electrical conductivity, showing great potential for high-temperature electrode applications. 